\title{
BIOMETRIC FOETAL CONTOUR EXTRACTION USING HYBRID LEVEL SET
}

\author{
Rachana Jaiswal and Srikant Satarkar \\ Department of Computer Science and Engineering, \\ Amravati University, Akola, MH, India
}

\begin{abstract}
In medical imaging, accurate anatomical structure extraction is important for diagnosis and therapeutic interventional planning. So, for easier, quicker and accurate diagnosis of medical images, image processing technologies may be employed in analysis and feature extraction of medical images. In this paper, some modifications to level set algorithm are made and modified algorithm is used for extracting contour of foetal objects in an image. The proposed approach is applied on foetal ultrasound images. In traditional approach, foetal parameters are extracted manually from ultrasound images. Due to lack of consistency and accuracy of manual measurements, an automatic technique is highly desirable to obtain foetal biometric measurements. This proposed approach is based on global \& local region information for foetal contour extraction from ultrasonic images. The primary goal of this research is to provide a new methodology to aid the analysis and feature extraction from foetal images.
\end{abstract}

\section{KEYWORDS}

Active contour, Region-based, Edge-based, Hybrid Approach, Foetal images.

\section{INTRODUCTION}

Image segmentation plays significant role in medical image processing and computer vision. It permits visualization of organs and structures in the body, analysis of the shapes and sizes of these organs to clinicians, and diagnosis of pathologies more quickly and accurately. Physical segmentation is a tiresome, time consuming process and most of the times it is not accurate. It is subjected to high variations from one expert to another due to its dependence on physician's subjective knowledge and his experience. The information generated from the computerized analysis of medical images can be used as a second opinion in making diagnostic decisions by physicians.

Foetal biometric ultrasound measurements pose an interesting problem due to presence of inhomogeneous intensity profile, strong speckle noise and shadows making it difficult to properly segment the correct positions and shapes of interesting regions. In addition to this, edges are poorly defined and are close to other foetal structures making segmentation task difficult using standard active contour models. The proposed work aims at developing a novel segmentation technique to extract foetal contour accurately with reduced dependence on initial curve placement [9]. 


\section{IMAge Segmentation TeChNiQue}

There are two approaches to segmentation similarity-based and discontinuity-based. In similaritybased approach, image is partitioned based on uniformity in intensity and texture. These methods create more coherent regions compared to discontinuity-based methods. In this approach, segmentation is performed based on the grey value of pixels without taking account of connectivity property. Thresholding, Region growing methods fall into this category. Discontinuity-based approach partitions image based on sudden intensity changes. At the point of intersection of two regions, intensities vary. In images, object boundaries represent edges. The traditional methods employ Sobel, Robert and Prewitt operators for edge detection. Another discontinuity-based approach involves use of deformable model.

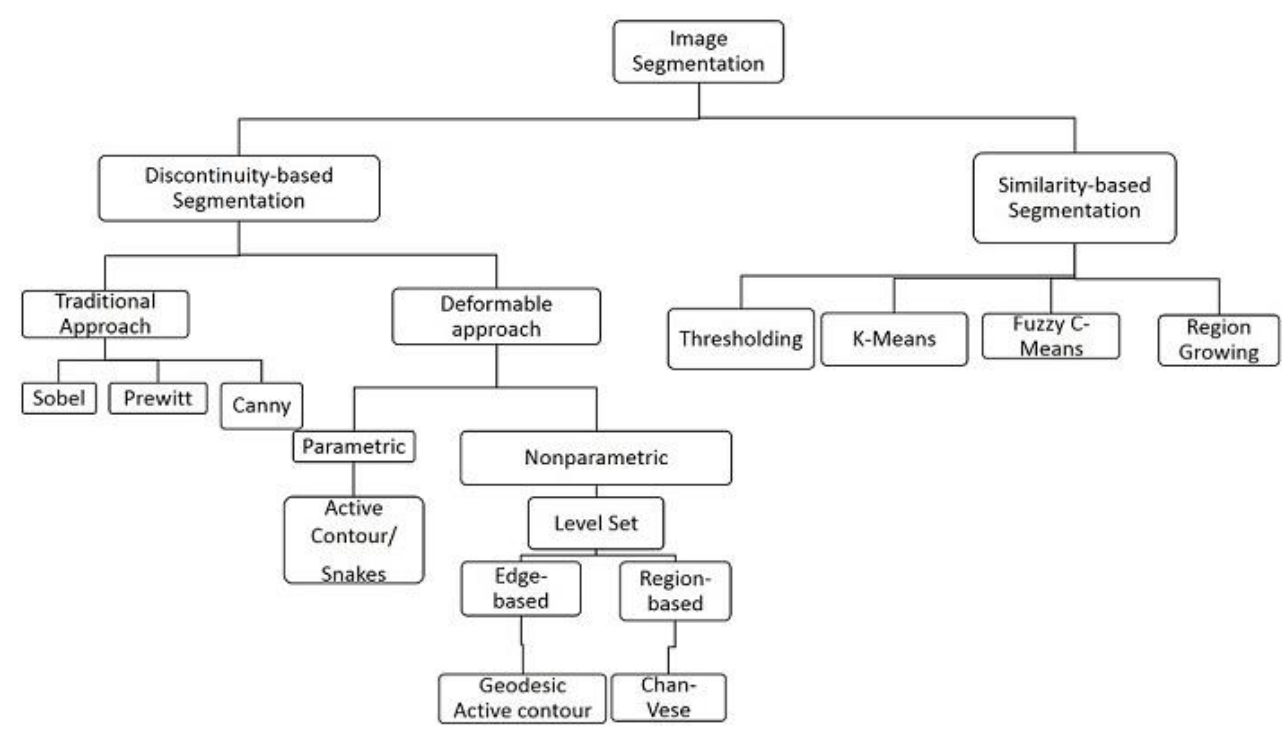

Figure 1: Image Segmentation Algorithm classification

Deformable models possess ability to directly generate closed parametric curves or surfaces from images and are robust to noise and spurious edges [2]. Deformable models can be categorized into the parametric deformable models and the geometric model depending on way of evolution.

Table 1: Comparison of parametric and nonparametric methods

\begin{tabular}{|l|l|}
\hline Parametric & Non-parametric/geometric \\
\hline $\begin{array}{l}\text { use explicit representation based on } \\
\text { Lagrange formulation }\end{array}$ & $\begin{array}{l}\text { represents the contour implicitly and } \\
\text { evolves according to the Euler formulation }\end{array}$ \\
\hline Initialization sensitive & $\begin{array}{l}\text { Initialization sensitivity is less compared to } \\
\text { parametric ones. }\end{array}$ \\
\hline Fail to converge to concavities & Affected by weak edges \\
\hline Top-down approach & Multiple boundaries at a time can be found. \\
\hline Prior knowledge can be easily incorporated & Are able to handle high curvature regions. \\
\hline Less human intervention. & $\begin{array}{l}\text { reduces the time required for image } \\
\text { analysis and subjectivity associated with } \\
\text { manual delineations and measurements [6]. }\end{array}$ \\
\hline Prior boundary information is available. & No prior information is available. \\
\hline
\end{tabular}




\section{IMAGE SEgmentation BASEd ON LEVEl SET}

It is proposed by Stanley Osher and James Sethian. Prior information regarding shape and the initial locations of the region to be segmented is not needed in this approach. It is widely used for medical, satellite and natural image segmentation, analysis and boundary extraction. It can handle corner, inflexion and topological structure changes and can also segment regions with irregular shape. It is initialization insensitive [3]. It can segment objects with deep concavities and can detect multiple objects [4].

Any segmentation problem can be solved by converting it into a PDE framework and employing finite difference methods. Let $\mathrm{C}$ represents contour evolving by the time $\mathrm{t}$ in the normal vector direction $\mathrm{N}$ with the speed function $\mathrm{F}(\mathrm{K})$ which depend on the curvature of the contour. The contour deformation using PDE can be written as,

$$
\frac{\partial c}{\partial t}=F(K) . N
$$

In a high curvature area, contour propagation speed is higher compared to a low curvature area [8]. In level set method, speed and direction of the propagation depends on image intensity gradient and the curvature. Internal and external energies along the boundary of curve are minimized by deforming the curve along its shape [11].

For a closed curve $\mathrm{C}$ which is dividing plane into two regions, signed distance function (SDF) is given $\phi(x, y)$. For point lying inside the curve $\mathrm{C}, \phi(x, y)>0$; for outside point, $\phi(x, y)<0 ; \phi(x$, $y)=0$ for the point lying on $\mathrm{C}$ [7]. To deal with topological changes naturally following equation is used:

$$
\frac{\partial \emptyset}{\partial t}+F(k)|\nabla \emptyset|=0
$$

It is not able to detect objects in images with low-contrast boundaries [5]. The geometric active contour models are classified into edge-based and region-based models. Edge-based active contour models use local edge information for curve evolution whereas region-based active contour models use statistical information. In traditional level set method, the leaking problem appears due to presence of a fuzzy or discrete boundary in the region. The iteration time is affected by too large or too small initial contour [13].

Table 2: Edge-based and Region-based Comparison

\begin{tabular}{|l|l|}
\hline Edge-based & Region-based \\
\hline $\begin{array}{l}\text { an edge stopping function is based on image } \\
\text { gradient. }\end{array}$ & $\begin{array}{l}\text { the motion of the contour relies on region } \\
\text { descriptor }\end{array}$ \\
\hline $\begin{array}{l}\text { Highly sensitive to noise, affected by fuzzy } \\
\text { or blurred boundaries. }\end{array}$ & $\begin{array}{l}\text { Insensitive to noise. It is less affected by } \\
\text { fuzzy or blurred boundaries. }\end{array}$ \\
\hline $\begin{array}{l}\text { Affected by initial curve placement. } \\
\text { Can segment heterogeneous objects }\end{array}$ & $\begin{array}{l}\text { robustness against initial curve placement. } \\
\text { not ideal for segmenting heterogeneous } \\
\text { objects. }\end{array}$ \\
\hline Uses local edge information & Uses statistical region information \\
\hline $\begin{array}{l}\text { Have high segmentation precision since it } \\
\text { allows user to mark boundary directly. }\end{array}$ & $\begin{array}{l}\text { Can detect interior and exterior boundaries } \\
\text { simultaneously. }\end{array}$ \\
\hline
\end{tabular}




\section{LeVEL SET IMAgE SEgMENTATION IMPROVEMENT}

Motivated by the work of [17], a hybrid segmentation approach which aims to blend the benefits of global and modified local region-based approaches to improve the quality of segmentation is proposed. It is a two-stage method which uses local and global intensity information.

Region-based level set approach is classified into global and local region-based approach. Initially, contour for foetal image is specified and global region-based approach is applied to it. Global approach is fast and stable with homogeneous regions. But our foetal images contain inhomogeneous regions and contour that is extracted is not proper. Hence, the output of this stage is given as input to modified local region-based approach which employs square window to give exact contour.

MATLAB is used for experimentation on personal computer with Intel Core i5, $1.7 \mathrm{GHz}$ and 4GB RAM. Sufficient medical images taken from authorized sonography centre are tested and after testing, result shows improvement in quality and efficiency of segmentation as compared to existing approaches. The parameters used for comparison of algorithms include Structural Similarity Index, Correlation, Mean Square Error, Peak Signal to Noise Ratio, Dice similarity coefficient, Jaccard Similarity Coefficient. The result of the evaluated values of Structural Similarity Index, Correlation, Mean Square Error, Peak Signal to Noise Ratio, Dice similarity coefficient, Jaccard Similarity Coefficient for some of the images are represented in comparison graph.

Proposed approach when tested over a large dataset of foetal images has given encouraging results. The results which are presented shows Structural Similarity index for different images using proposed approach performs $6.94 \%$ better over existing method, for Correlation it is $5.97 \%$, MSE is decreased by $66.06 \%$, Peak Signal to Noise ratio is improved by $16.73 \%$, for Dice Similarity Coefficient it is $5.9 \%$ and for Jaccard Similarity Coefficient it is $29.89 \%$. So, it is proposed after careful observations that the hybrid approach gives better performance among all other segmentation techniques.

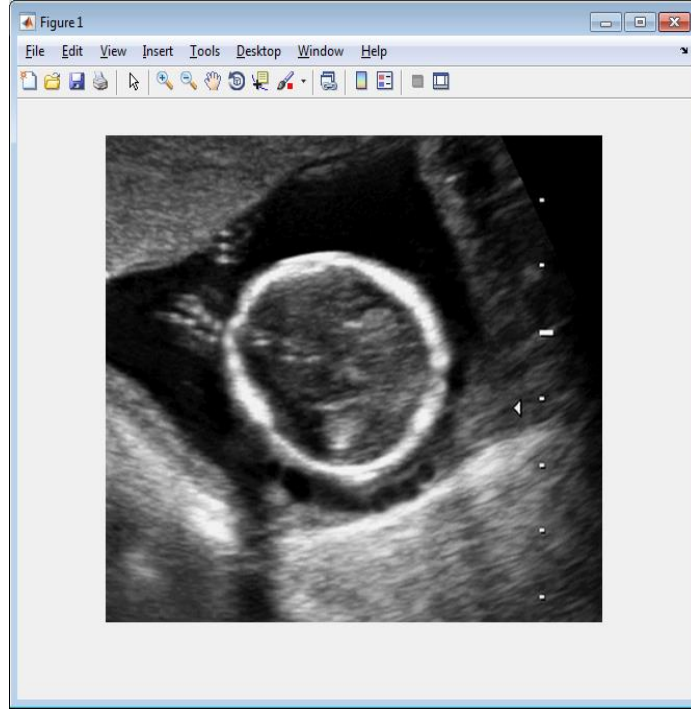

Figure 2: a) I ${ }^{\text {st }}$ Input Image

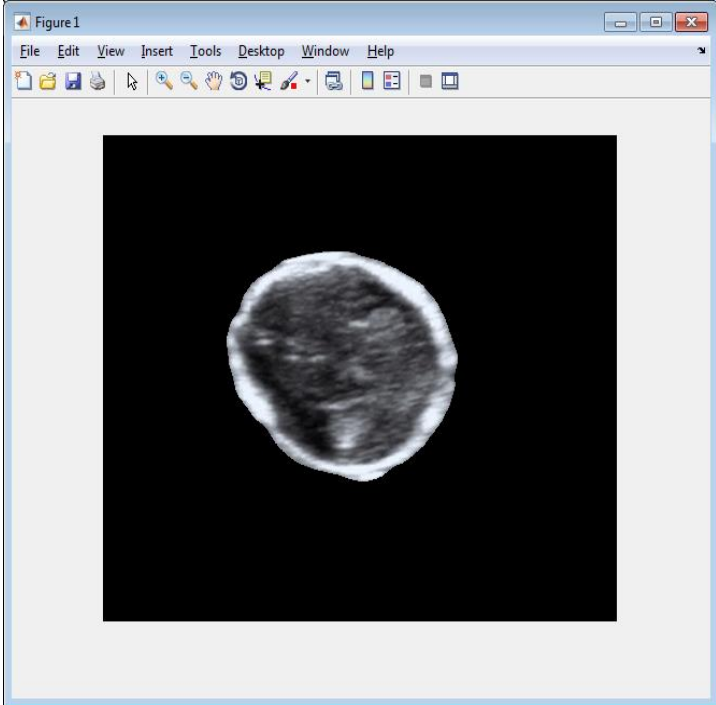

b) Result of Proposed Hybrid Method 


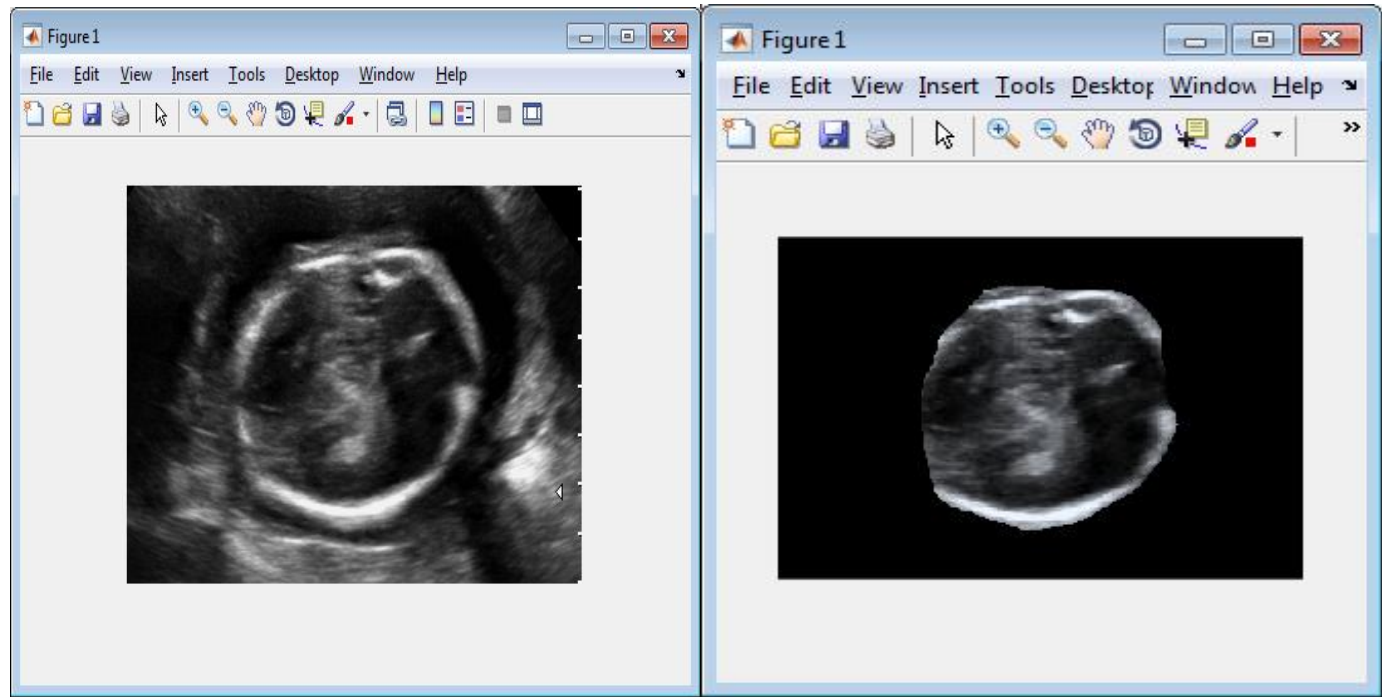

Figure 3: a) II ${ }^{\text {nd }}$ Input Image

b) Result of Proposed Hybrid Method

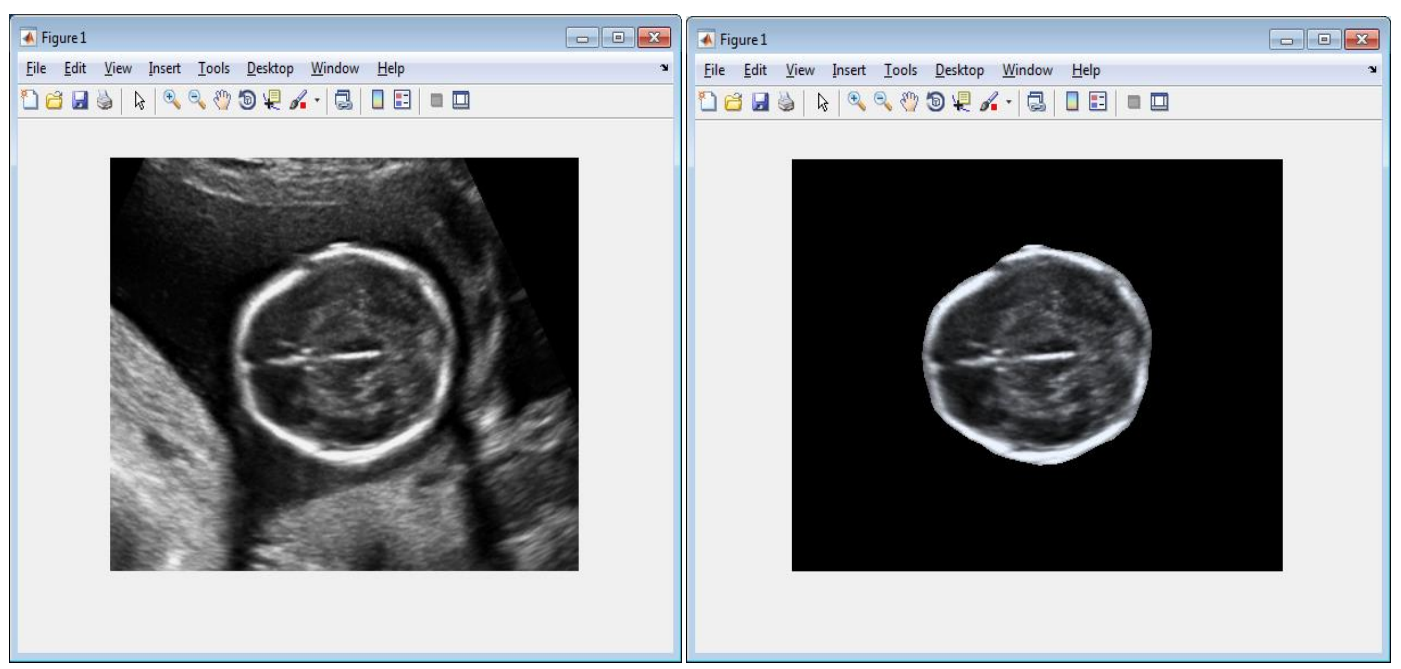

Figure 4: a) III ${ }^{\text {rd }}$ Input Image

b) Result of Proposed Hybrid Method

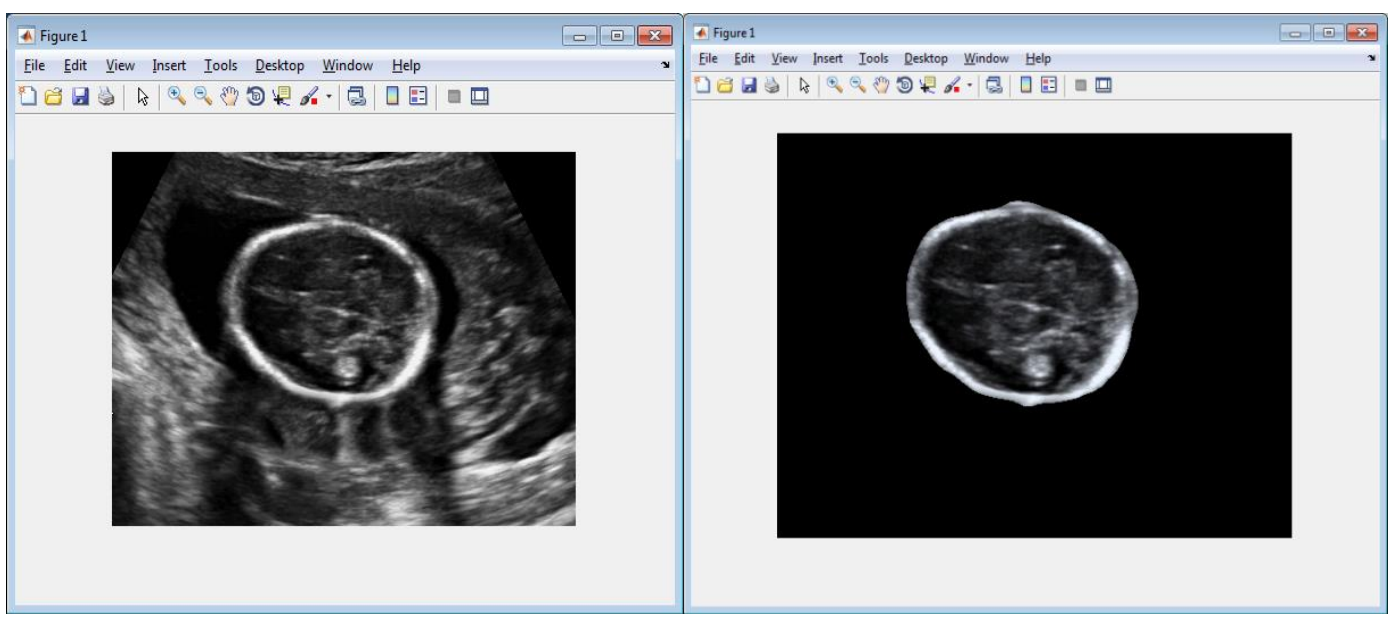

Figure 5: a) IV ${ }^{\text {th }}$ Input Image

b) Result of Proposed Hybrid Method 


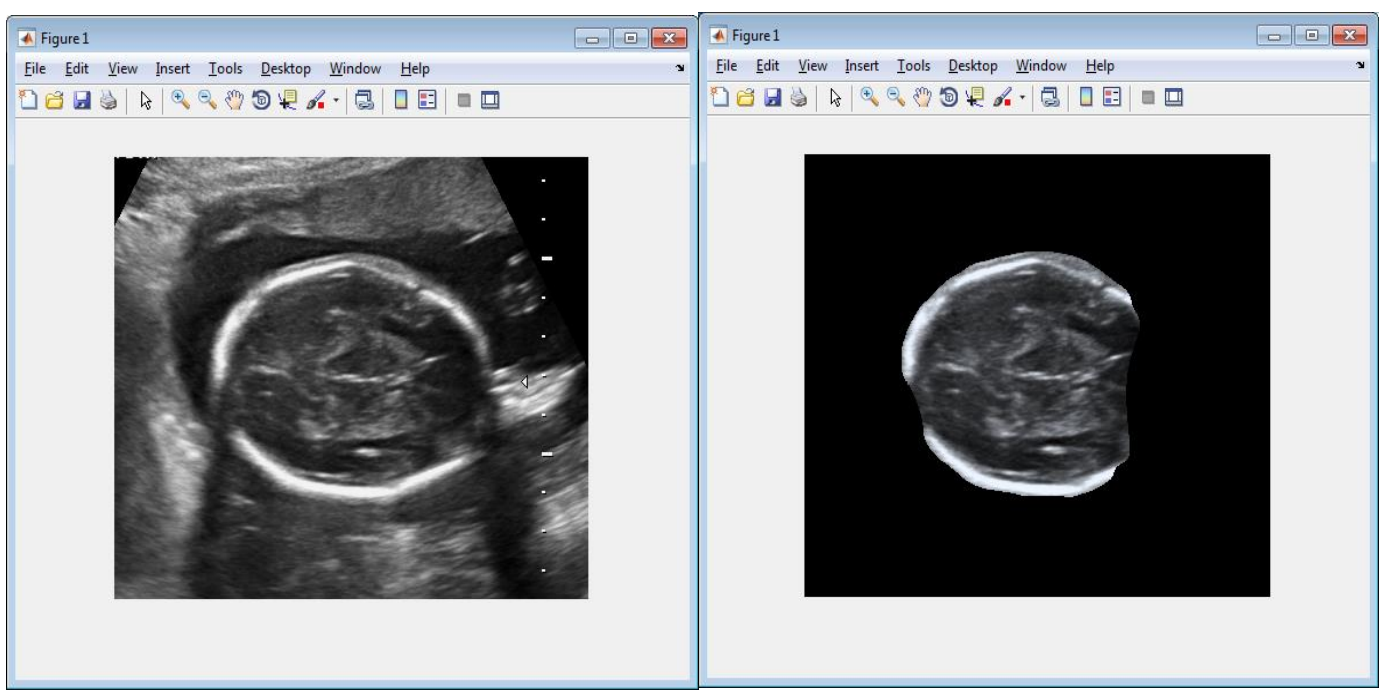

Figure 6: a) $\mathrm{V}^{\text {th }}$ Input Image

b) Result of Proposed Hybrid Method

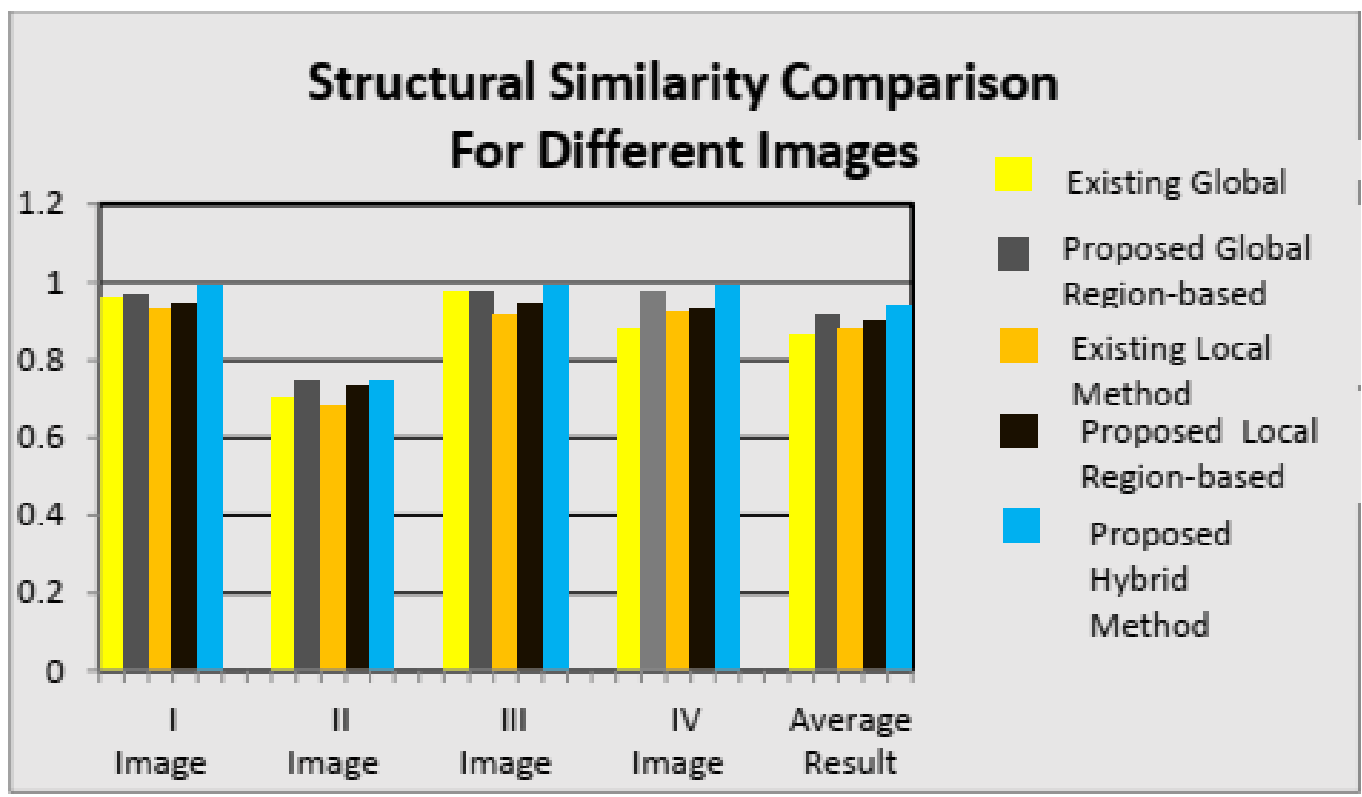

Figure 7: Structural Similarity Comparison for different images 


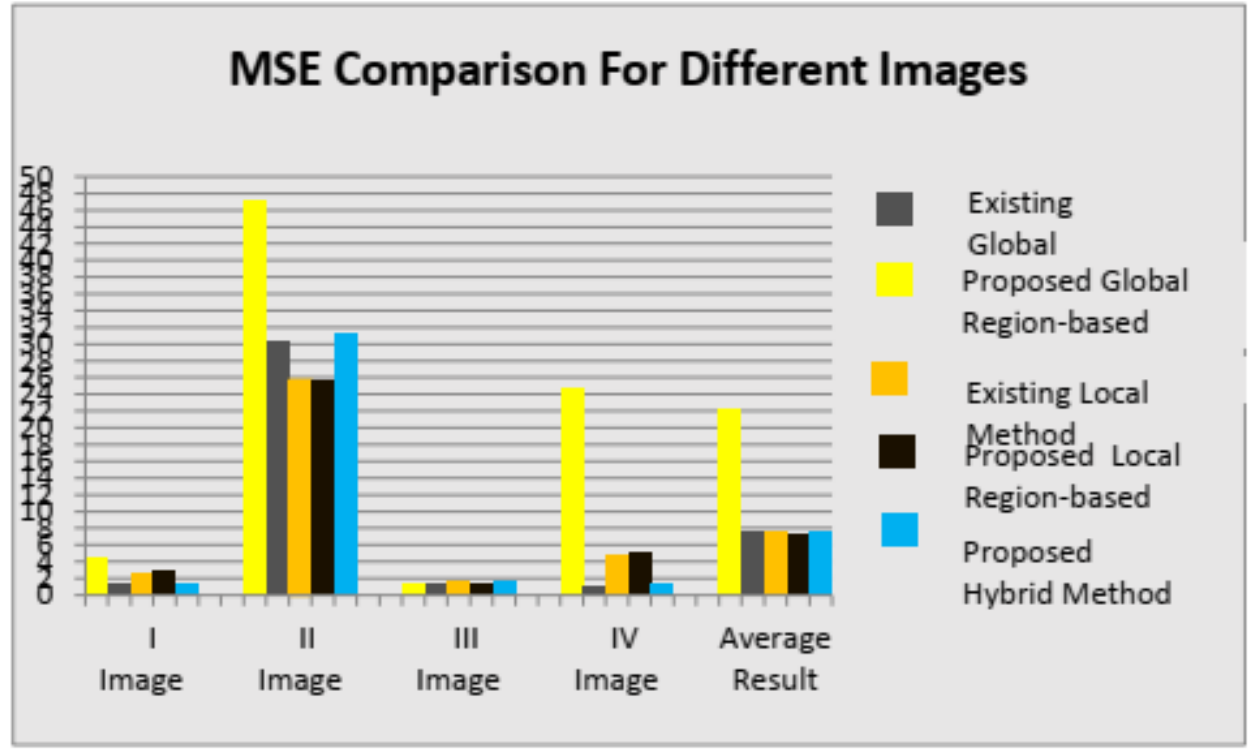

Figure 8: MSE Comparison for different images

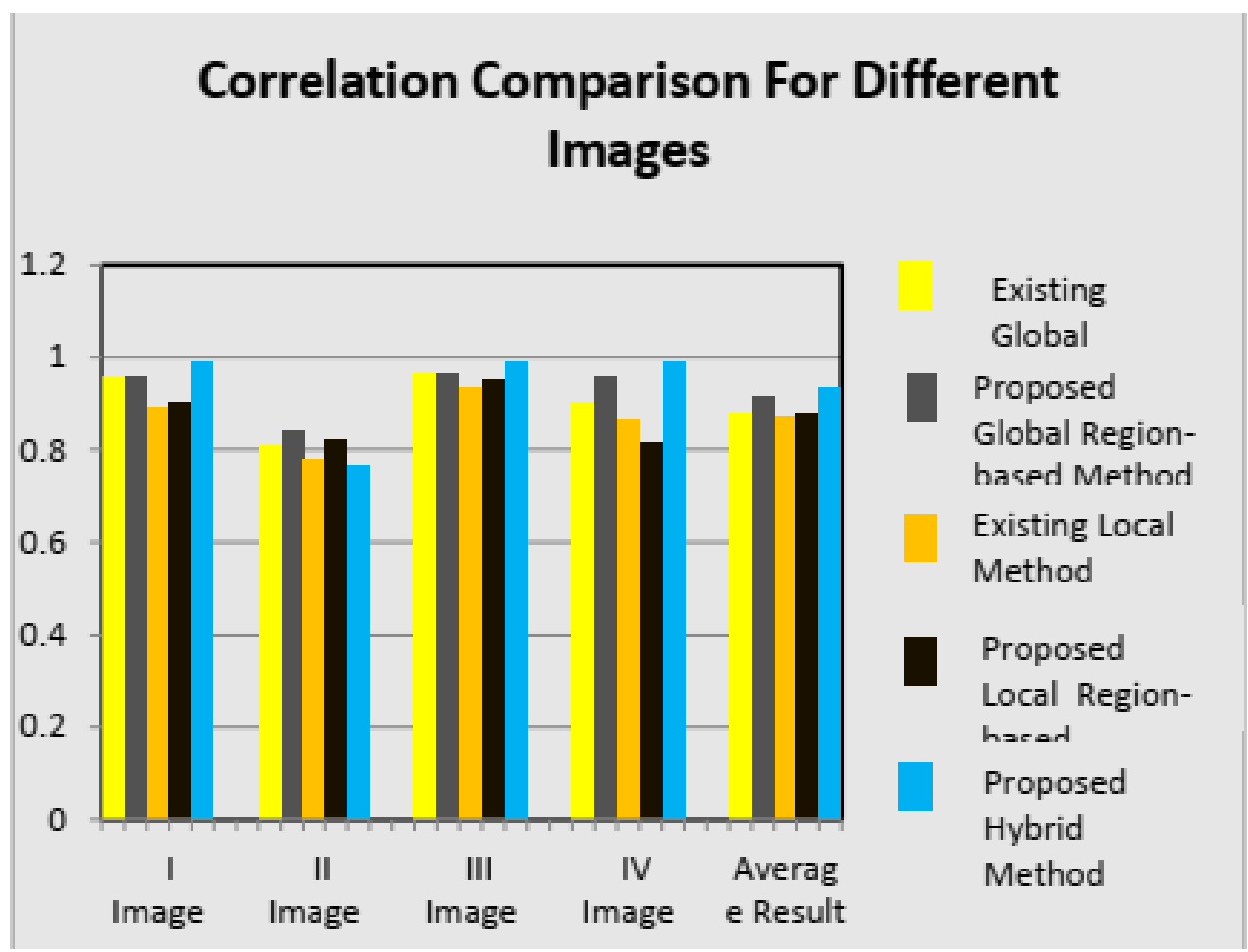

Figure 9: Correlation Comparison for different images 


\section{PSNR Comparison For Different Images}

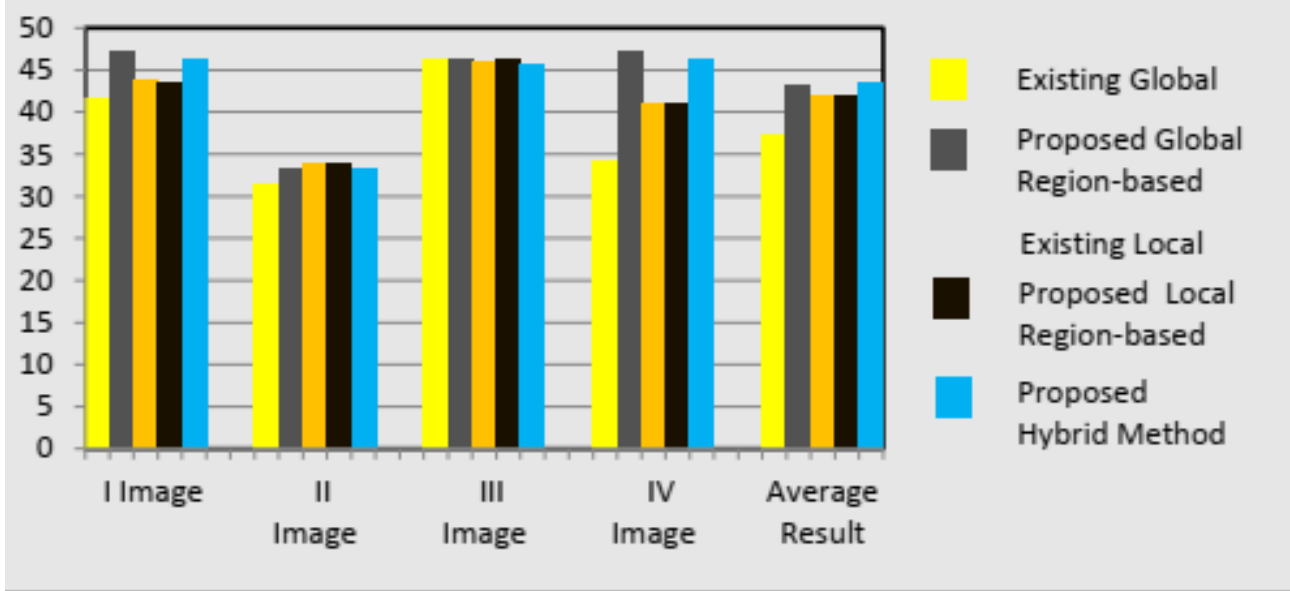

Figure 10: Peak Signal to Noise Ratio Comparison for different images

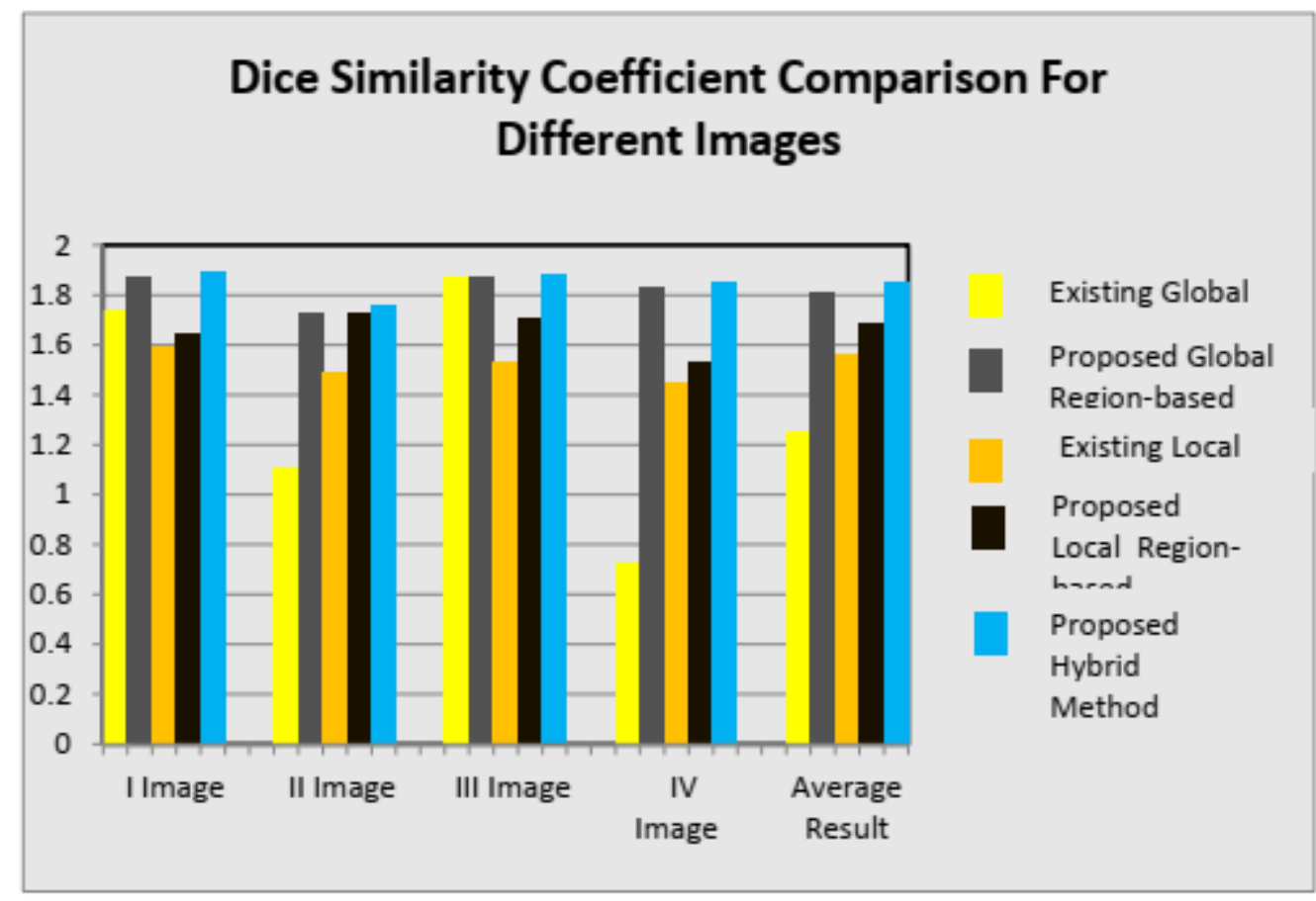

Figure 11: Dice Similarity Coefficient Comparison for different images 


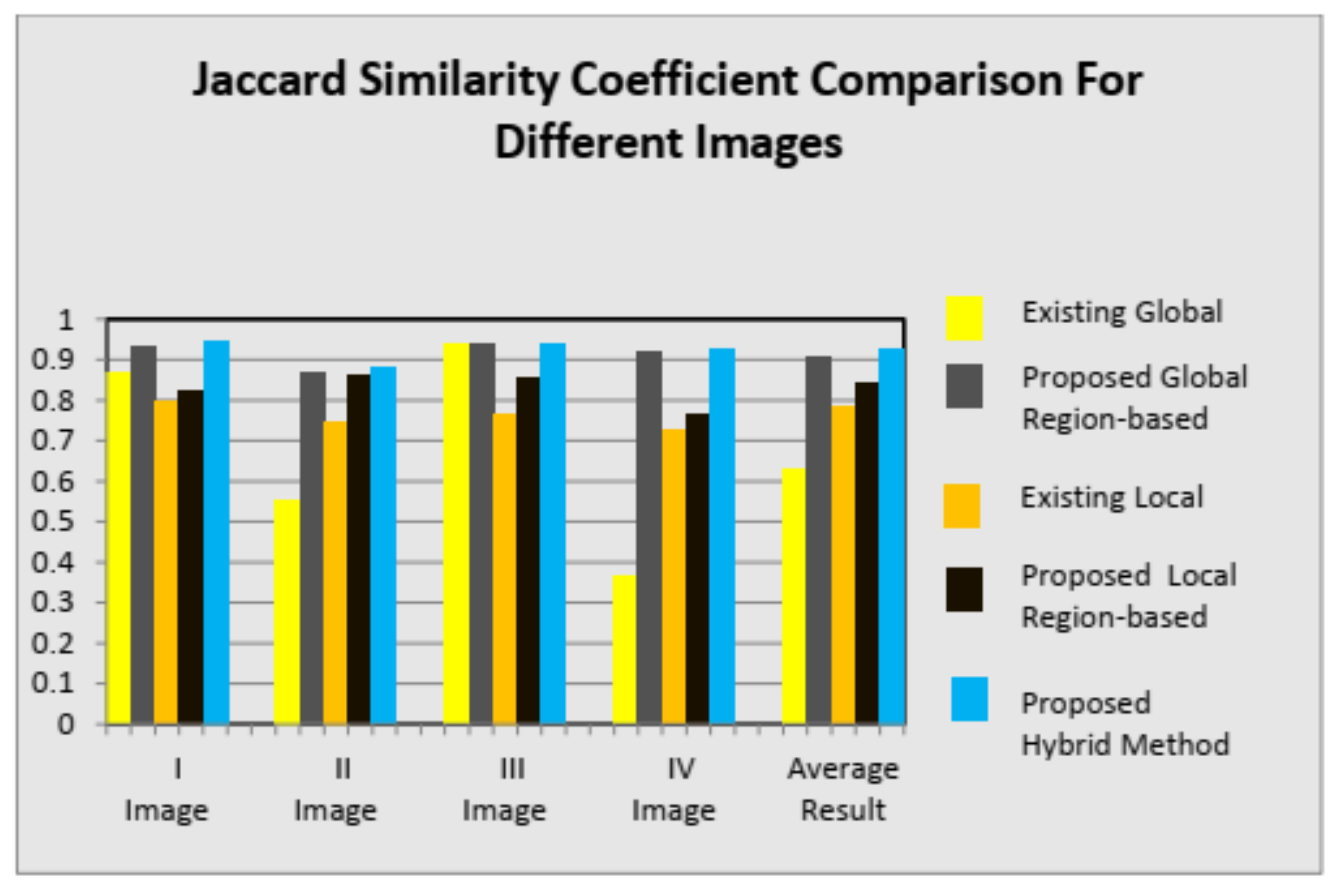

Figure 12: Jaccard Similarity Coefficient Comparison for different images

\section{CONClusion}

Perona and Malik smoothing technique is applied prior to segmentation [18]. The narrow band method is employed in the proposed approach. The proposed global region-based model is insensitive to the location of initial contour. The model could not produce correct result in presence of heterogeneous object. So, after observing the performance of global region-based approach, local region-based approach is implemented. It is a local method, so in presence of heterogeneous object or incomplete edges, this algorithm produces correct result. In order to obtain benefits of both methods, result after application of proposed global method which is rough segmentation is given as input to proposed local method for suitable initialization. The segmentation method is robust to different types of initialization and provides more accurate foetal contour which can be used for further analysis.

\section{REFERENCES}

[1] Zhou Wang, Alan C. Bovik, Hamid R. Sheikh and Eero P. Simoncelli, (2004) "Image quality assessment: From error visibility to structural similarity," IEEE Transactions on Image Processing, pp.600-612.

[2] HaiJun Wang, Ming Liu and WenLai Ma, (2010) "Color Image Segmentation Based on a New Geometric Active Contour Model", International Conference on Machine Vision and Humanmachine Interface, DOI: 10.1109/MVHI.2010.75, pp. 6 - 9.

[3] Mohamed Ali Charmi, Mohamed Amine Mezghich, Slim M'Hiria, Stephane Derrode and Faouzi Ghorbel, (2010) "Geometric shape prior to region-based active contours usingFourier-based shape alignment", IEEE International Conference on Imaging Systems and Techniques, DOI: 10.1109/IST.2010.5548489, pp.478 - 481, 2010.

[4] Yun-Jen Chiu, Van-Truong Pham, Thi-Thao Tran and Kuo-Kai Shyu,(2010) "Evaluation of active contour on medical inhomogeneous image segmentation", $3^{\text {rd }}$ International Conference on Computer Science and Information Technology, DOI:10.1109/ICCSIT.2010.5564101,pp.311 - 314, 2010. 
[5] Y.Ebrahimdoost, J.Dehmeshki, T.S.Ellis, M.Firoozbakht, A.Youannic and SD.Qanadli,(2010) "Medical Image Segmentation Using Active Contours and a Level Set Model: Application to Pulmonary Embolism (PE) Segmentation”, IEEE Xplore, DOI: 10.1109/ICDS.2010.64, pp.269 - 273, 2010.

[6] Jieyu Cheng, Mingyue Ding and Xuming Zhang, (2011) "Geometric Active Contour Model and its Application to Carotid Plaque Detection", International Conference on Intelligent Computation and Bio-Medical Instrumentation, DOI:10.1109/ICBMI.2011.62, pp.101 - 104, 2011.

[7] Jinping Fan, Ruichun Wang, Shiguo Li and Chunxiao Zhang, (2012) "Automated cervical cell image segmentation using level set based active contour model", in 12th International Conference on Control Automation Robotics \& Vision (ICARCV), DOI: 10.1109/ICARCV.2012.6485273, pp.877 882, 2012.

[8] Evgin Goceri, Mehmet Z. Unlu, Cuneyt Guzelis and Oguz Dicle, (2012) "An automatic level set based liver segmentation from MRI data sets", 3rd International Conference on Image Processing Theory, Tools and Applications (IPTA), DOI:10.1109/IPTA.2012.6469551, pp.192 - 197, 2012.

[9] Amit Satish Unde, V. A. Premprakash and Praveen Sankaran, (2012) "A novel edge detection approach on active contour for tumor segmentation", Students Conference on Engineering and Systems, DOI: 10.1109/SCES.2012.6199100, pp.1-6, 2012.

[10] Xie Li-Zhi, Tian Yun, Zhou Ming-Quan and Cao Rong-Fei,(2012) "Cerebrovascular segmentation based on region growing and level set algorithm", International Conference on Audio, Language and Image Processing, DOI: 10.1109/ICALIP.2012.6376733,pp.854 - 859, 2012.

[11] Hassan Mouhadjer, M. Mansour, M. Ouslim and B. Bouchiba, (2013) "Segmentation of human chromosome images using distance regularized level set evolution", $2^{\text {nd }}$ International Conference on Advances in Biomedical Engineering, DOI:10.1109/ICABME.2013.6648 886, pp.215 - 218, 2013.

[12] Dinesh Reddy Byreddy and MV Raghunadh, (2014) "An application of geometric active contour in bio-medical engineering", International Conference on Circuits, Systems, Communication and Information Technology Applications (CSCITA), DOI: 10.1109/CSCITA.2014.6839280, pp.322 326, 2014.

[13] Mengjuan Chen, Jianwei Li, Hanqing Zhao and Xiao Ma, (2014) "A local region-based level set algorithm for image segmentation", IEEE Workshop on Electronics, Computer and Applications, DOI: 10.1109/IWECA.2014.6845753, pp.844 - 847, 2014.

[14] Zhiwei Liu, Dongao Zhou, Qiang Lin and Jiayu Lin, (2015) "Active Contour Model Based on Local and Global Image Information", 7th International Conference on Intelligent Human-Machine Systems and Cybernetics, DOI: 10.1109/IHMSC.2015.63, pp.266 - 269, 2015.

[15] Snehal Kulkarni and Sanjay Shitole, (2016) "Region growing based novel approach for estimation of speckle in SAR images", IEEE Bombay Section Symposium (IBSS), DOI: 10.1109/IBSS.2016. 7940 200, pp.1-5, 2016.

[16] Harmeet Saini and Varsha Sahni, (2017) "Region Growing segmentation using de-noising algorithm for medical ultrasound images", 3rd International Conference on Computational Intelligence \& Communication Technology (CICT), DOI: 10.1109/CIACT.2017 .7977 313, pp. 1 - 5, 2017.

[17] Soomro S, Munir A and Choi KN, "Hybrid two-stage active contour method with region and edge information for intensity inhomogeneous image segmentation", PLOS ONE 13(1): e0191827, [Online]. Available: https://doi.org/10.1371/ journal.pone. 0191827.

[18] Shafiullah Soomro and Kwang Nam Choi, (2018) "Active Contours Based on An Anisotropic Diffusion", Digital Image Computing: Techniques and Applications (DICTA), DOI: 10.1109/DICTA.2018.8615767, pp. 1 - 6, 2018.

(C) 2020 By AIRCC Publishing Corporation. This article is published under the Creative Commons Attribution (CC BY) license. 